\title{
Sizing Methodology based on Scaling Laws for a Permanent Magnet Electrical Variable Transmission
}

\author{
Florian Verbelen, Ahmed Abdallh, Hendrik Vansompel, Kurt Stockman, Peter Sergeant
}

\begin{abstract}
This paper discusses a method to design an electrical variable transmission of which the stator and inner rotor contain a distributed three-phase winding, and the outer rotor is equipped with permanent magnets and a DC-field winding. The main problem with the design of electrical variable transmissions is that the modeling relies on time-consuming finite element calculations. Studying the effect of design changes is, therefore, a cumbersome task. To minimize the need for finite element calculations, scaling laws are applied, which reduce the computational effort to a matter of milliseconds per design. Therefore, the losses of multiple scaled designs can be analyzed for a given load cycle while taking constraints into account such as maximum torque. By presenting this data in a performance map, the optimal design regarding the losses is easily deduced. To validate the presented methodology, finite element calculations, measurements and available literature are used.
\end{abstract}

Index Terms-Design methodology, Energy efficiency, Electrical Variable Transmission, Permanent magnet machines, Scaling law

\section{INTRODUCTION}

In the past decade, many researchers in academia and industry have studied ways to develop variable transmissions. These torque converters can vary the speed ratio between the input and output shaft in a continuous way. This feature has been proven to be useful in wind power generation systems [1], [2] as well as in vehicle applications [3].

Until now, the mechanical variable transmission, also known as Continuously Variable Transmission (CVT) has received more attention from industry as the idea behind the technology is much more mature. However, in the past years a shift in interest towards the Electrical Variable Transmission (EVT) is noticeable. The main reason for that shift is the higher efficiency of the EVT throughout the operating range as

Manuscript received September 18, 2018; revised December 6, 2018; accepted February 25, 2019. This work was carried out for the EMTechno project (project ID: IWT150513) supported by VLAIO and Flanders Make, the strategic research center for the manufacturing industry.

Electrical Energy Laboratory, Department of Electrical Energy, Metals, Mechanical Constructions and Systems, Ghent University, 9000 Ghent, Belgium, and also with Flanders Make, 3920 Lommel, Belgium (email: Florian.Verbelen@UGent.be; Hendrik.Vansompel@UGent.be; Ahmed.Abdallh@Flandersmake.be; Kurt.Stockman@UGent.be; Peter.Sergeant@UGent.be).
TABLE I

NOMENCLATURE

$\begin{array}{ll}T_{\mathrm{r} 1} & \text { inner rotor torque } \\ T_{\mathrm{r} 2} & \text { outer rotor torque } \\ T_{\mathrm{s}} & \text { stator torque } \\ N_{\mathrm{r} 1} & \text { inner rotor speed } \\ N_{\mathrm{r} 2} & \text { outer rotor speed } \\ I_{\mathrm{sq}} & \text { current in the stator (q-axis) } \\ I_{\mathrm{sd}} & \text { current in the stator (d-axis) } \\ I_{\mathrm{r} 2 \mathrm{~d}} & \text { current in the DC-field winding } \\ I_{\mathrm{r} 1 \mathrm{q}} & \text { current in the inner rotor (q-axis) } \\ I_{\mathrm{r} 1 \mathrm{~d}} & \text { current in the inner rotor (d-axis) } \\ \Psi_{\mathrm{sq}} & \text { stator flux (q-axis) } \\ \Psi_{\mathrm{sd}} & \text { stator flux (d-axis) } \\ \Psi_{\mathrm{r} 1 \mathrm{q}} & \text { inner rotor flux (q-axis) } \\ \Psi_{\mathrm{r} 1 \mathrm{~d}} & \text { inner rotor flux (d-axis) } \\ K_{\mathrm{A}} & \text { axial scaling factor } \\ K_{\mathrm{R}} & \text { radial scaling factor } \\ K_{\mathrm{W}} & \text { winding scaling factor } \\ K_{\mathrm{J}} & \text { current density scaling factor } \\ a_{\mathrm{p}} & \text { number of parallel paths } \\ N_{\mathrm{c}} & \text { number of turns per coil } \\ l_{\mathrm{a}} & \text { active length of the EVT } \\ D & \text { diameter of the EVT } \\ A_{\mathrm{z}} & \text { cross-sectional surface of the EVT } \\ P_{\mathrm{loss}} & \text { power losses } \\ P_{\mathrm{cu}} & \text { copper losses } \\ P_{\mathrm{fe}} & \text { iron losses } \\ F_{\mathrm{d}} & \text { force density } \\ T_{\mathrm{m} \text { max }} & \text { maximum torque } \\ R_{\mathrm{r} 1} & \text { resistance of the inner rotor } \\ R_{\mathrm{r} 2} & \text { resistance of the outer rotor } \\ R_{\mathrm{S}} & \text { resistance of the stator } \\ \hat{B} & \text { amplitude of the magnetic flux density } \\ f & \text { frequency } \\ \text { subscript } 0 & \text { refers to the reference design } \\ \text { superscript s } & \text { refers to scaled properties } \\ & \end{array}$

demonstrated in [4], [5]. Furthermore, reduced maintenance and faster dynamics are considered important advantages.

As with CVTs, many different topologies for EVT exist of which in this paper a Permanent Magnet (PM) version is used as described in [6]. The design process of any EVT offers many challenges due to the many degrees of freedom. In [7] the optimal stator-slot/outer-rotor-pole/inner-rotor-slot number has been determined for a synchronous PM-EVT. The optimal combination was distinguished by low voltage harmonics in the back-EMF, high average torque with low ripple and low iron loss. In [8], changes in height, span and position of the PM material are considered together with changes in PM layers and shapes. Cheng [9] applied a system level approach where the overall dimensions for the PM-EVT are defined according 
to the main design equations for a normal, i.e. one rotor, PM synchronous machine (PMSM) as formulated in [10].

The methods proposed in [7]-[9] provide insight, to some extend, in design modifications but require a lot of Finite Element (FE) calculations. Such methods are accurate but rather time-consuming to determine the complex relation between current and flux.

To solve the issues with the FE simulations, scaling laws defined for a PMSM will be used as defined in [11] to optimize the general size of the EVT. A brief survey on scaling laws is given in section III. These scaling laws allow to scale a well-known EVT design, called later the reference design, of which all current to flux relations are known, in both axial and radial directions. In order to fit the voltage requirements of the scaled EVT with the power supply, a winding scaling factor can be used.

This ultra-fast scaling method will be combined with the validated optimal control of a PM-EVT [12] to obtain a method which can optimize the topology, in terms of copper and iron losses, in any given Operating Point (OP). This OP is defined by the torque developed by the inner rotor and stator and the speed of both rotors. The methodology is thus applicable for any kind of application. However, the method developed in this paper will most likely be used to optimize the drivetrain of a Hybrid Electrical Vehicle (HEV) equipped with an EVT. Similar optimization routines based on scaling laws developed for electrical vehicles with PMSM have already shown a great potential [13], [14].

The novelty of this paper is in applying the scaling law strategy to an EVT, not in the scaling law strategy itself as this has already been published in [11]. Furthermore, the scalable optimal control, which is used in this paper, is one of the main contributions. Finally, the combination of simultaneously optimizing geometry and control of a PM-EVT via scaling laws has, to the knowledge of the authors, not been presented in literature so far.

This paper is structured as follows. The reference EVT is shortly described in section II. In section III, the scaling laws for the EVT are discussed and validated based on FE results and available literature. Note that the FE results of the reference machine are validated through measurements on an experimental setup with fully controllable load speeds and torques, and fully controllable waveforms in the two power electronic converters of the EVT [6], [12], [15]. Based on the scaling laws, scalable optimal control is obtained as demonstrated in section IV. In section V, a design methodology is proposed which is based on the scaling laws. To demonstrate the work flow, a case study is considered in section VI. Finally, in section VII the conclusions of the research are formulated.

\section{Reference EVT}

As mentioned in the introduction, a well-known design of a PM-EVT will be used as a reference EVT of which the main machine parameters are given in Table II. Fig. 1 shows a partial cross-sectional view of this reference EVT. In this machine, both the stator and inner rotor contain a distributed three-phase winding (denoted by a, b and c). Validation of the
TABLE II

TABLE WITH MACHINE PARAMETERS.

\begin{tabular}{|l|c|c|c|}
\cline { 2 - 4 } \multicolumn{1}{c|}{} & stator & outer rotor & inner rotor \\
\hline rated power [kW] & $77^{*}$ & $120^{* *}$ & $75^{* *}$ \\
\hline rated current amplitude [A] & 265 & $4.6(\mathrm{DC})$ & 150 \\
\hline continuous torque [Nm] & 245 & 382 & 137 \\
\hline number of pole pairs & 4 & 4 & 4 \\
\hline outer radius [mm] & 175 & 123.5 & 102 \\
\hline inner radius [mm] & 124.5 & 103 & 57 \\
\hline PM volume [mm $\left.{ }^{2}\right]$ & - & 206200 & - \\
\hline active axial length [mm] & 87 & 87 & 87 \\
\hline winding resistance $[\Omega]$ & 0.0221 & 12.73 & 0.0256 \\
\hline DC-bus voltage [V] & 500 & - & 500 \\
\hline$*$ airgap power & \multicolumn{3}{|c}{} \\
** mechanical power &
\end{tabular}

stator back-EMF $E_{\mathrm{a}}$ of phase a is given in Fig. 2 (a). On the outer rotor, a single layer of permanent magnets is combined with a DC-field winding. When the absolute value of the DCfield current increases, the magnetic state of the flux bridge located underneath the DC-field winding changes. The purpose of this DC-field winding is thus to modify the path of the permanent magnet flux which changes the stator flux linkage. The inner rotor field is almost not affected by the DC-field current since the magnets have a low recoil permeability of about 1.05 [6]. Using the DC-field current, it is thus possible to change the stator flux linkage, while maintaining the rotor flux linkage which is particularly useful in HEV applications [6], [16]. In such applications the EVT can be used as CVT as well [5], where the battery power is kept zero. The power factor of the stator side during CVT-mode is presented in Fig. 2 (b). When the speed ratio $\tau$ equals 1 , inner and outer rotor torque are equal. The stator torque is thus, regarding to Newtons third law, equal to zero. As a consequence, the stator $d$-axis and $q$ axis currents are zero which explains the low power factor when the speed ratio is 1 . However, a good correspondence is noticed between the measurements and the FE results.

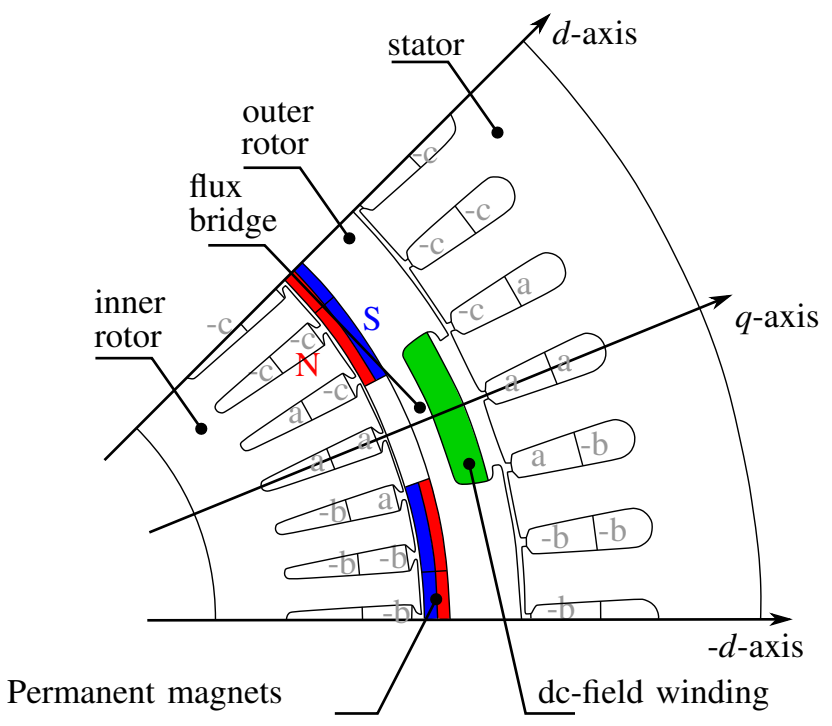

Fig. 1. Cross-sectional view of the EVT considered [15].

The stator torque $T_{\mathrm{s}}$ and inner rotor torque $T_{\mathrm{r} 1}$ are calculated according to (1) and (2) in which the stator and 


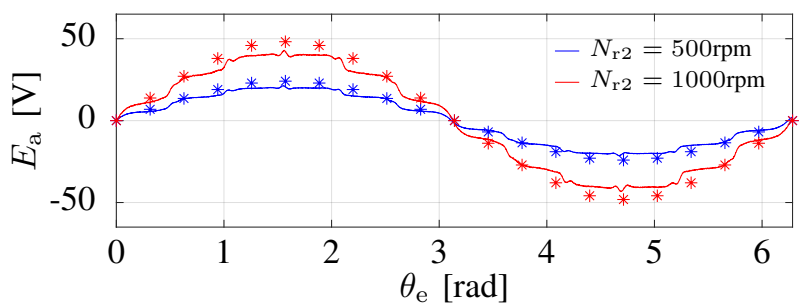

(a)

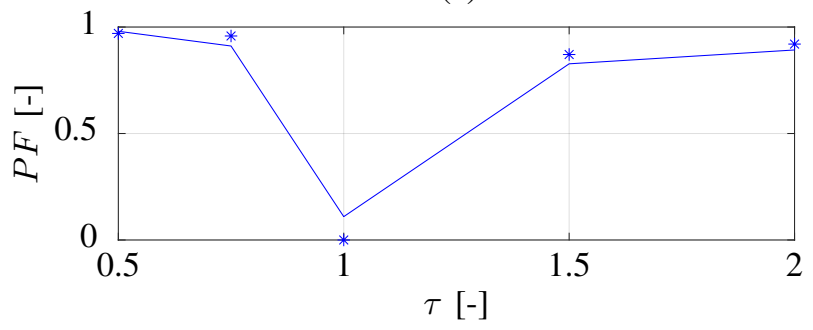

(b)

Fig. 2. (a) Back-EMF $E_{\mathrm{a}}$ of phase a as function of the electrical angular position $\theta_{\mathrm{e}}$ (b) Power factor $P F$ of the stator side for varying speed ratio $\tau=\frac{N_{\mathrm{r} 2}}{N_{\mathrm{r} 1}}$, input operating point is fixed at $T_{\mathrm{r} 1}=-40 \mathrm{Nm}$ and $N_{\mathrm{r} 1}=1000 \mathrm{rpm}$. Full lines are obtained via measurements while the stars are based on FE calculations. The considered EVT in this figure is the reference EVT.

inner rotor are denoted with subscript $s$ and $r 1$ respectively. Furthermore, flux $\Psi$ and current $I$ are related to the $d q$ reference frame (related to the outer rotor) and $N_{\mathrm{p}}$ is the number of pole pairs.

$$
\begin{gathered}
T_{\mathrm{s}}=\frac{3}{2} N_{\mathrm{p}}\left(\Psi_{\mathrm{sq}} I_{\mathrm{sd}}-\Psi_{\mathrm{sd}} I_{\mathrm{sq}}\right) \\
T_{\mathrm{r} 1}=\frac{3}{2} N_{\mathrm{p}}\left(\Psi_{\mathrm{r} 1 \mathrm{q}} I_{\mathrm{r} 1 \mathrm{~d}}-\Psi_{\mathrm{r} 1 \mathrm{~d}} I_{\mathrm{r} 1 \mathrm{q}}\right)
\end{gathered}
$$

The fluxes (stator flux in the $d$-axis $\Psi_{\text {sd }}$, stator flux in the $q$ axis $\Psi_{\mathrm{sq}}$, inner rotor flux in the $d$-axis $\Psi_{\mathrm{r} 1 \mathrm{~d}}$ and inner rotor flux in the $q$-axis $\Psi_{\mathrm{r} 1 \mathrm{q}}$ ) in (1) and (2) depend on 5 independent current setpoints: stator current in the $d$-axis $I_{\text {sd }}$, stator current in the $q$-axis $I_{\mathrm{sq}}$, current in the DC-field winding of the outer rotor $I_{\mathrm{r} 2 \mathrm{~d}}$, inner rotor current in the $d$-axis $I_{\mathrm{r} 1 \mathrm{~d}}$ and inner rotor current in the $q$-axis $I_{\mathrm{r} 1 \mathrm{q}}$. The relation between flux and current is determined based on FE calculations of which an example is given in Fig. 3 (full lines). The stars on Fig. 3 represent data based on measurements as validation of the FE model. A more in-depth validation of the generated torque and flux as a function of current can be found in [6].

As the flux linkage with each winding depends on the 5 different current components, a vast number of FE calculations is necessary to analyze the behavior of the EVT in every OP. However, not every current component has an even large impact on a flux component. The current in the DC-field winding has, for example, a significant impact on the $d$-axis flux in the stator (see Fig. 3 (a)) but a negligible impact on the $q$-axis flux in the stator (see Fig. 3 (b)). By applying this knowledge of how the flux is related to the current components, the number of FE calculations can be limited to 175000 . As one FE evaluation takes $2 \mathrm{~s}$, determining all current to flux relations takes several days per design, which makes the FE based approach impractical to study design changes (total simulation time can be decreased by using multiple processor cores). Therefore, a solution is presented in the next section based on scaling laws.

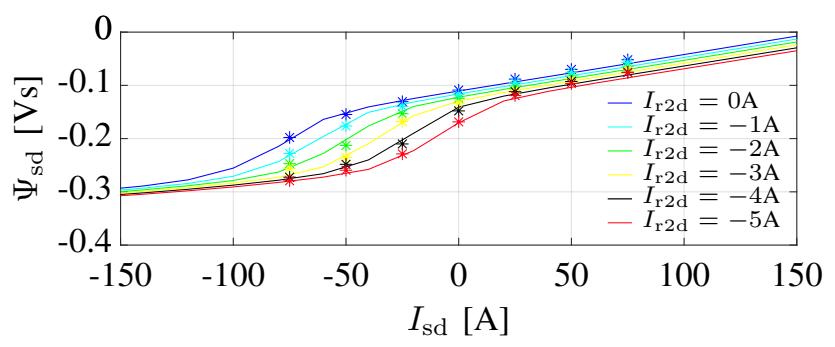

(a)

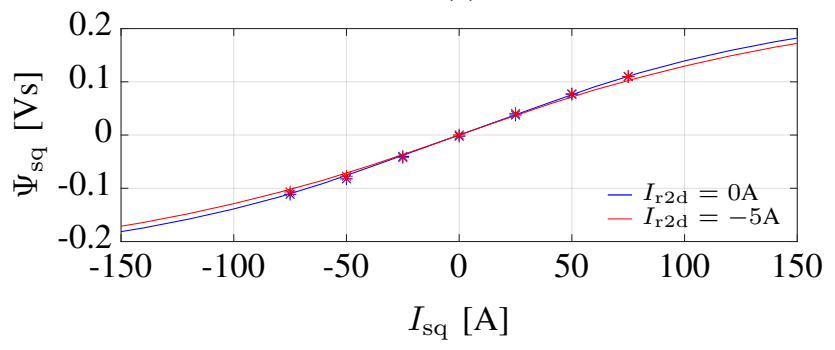

(b)

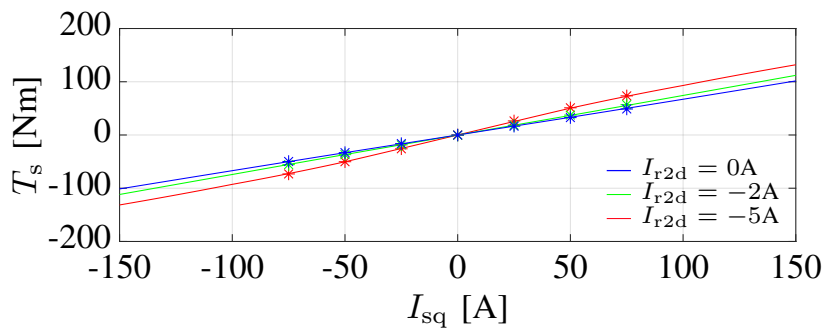

(c)

Fig. 3. (a) Stator flux in the $d$-axis $\Psi_{\text {sd }}$ as function of the stator current in the $d$-axis $I_{\mathrm{sd}}$ for varying DC-field current $I_{\mathrm{r} 2 \mathrm{~d}}$. The $d$-axis flux remains negative due to the chosen orientation of the $d$-axis (see Fig. 1). (b) Stator flux in the $q$-axis $\Psi_{\mathrm{sq}}$ as function of the stator current in the $q$-axis $I_{\mathrm{sq}}$ for varying DC-field current $I_{\mathrm{r} 2 \mathrm{~d}}$. (c) Stator torque $T_{\mathrm{s}}$ as function of stator current in the $q$-axis $I_{\mathrm{sq}}$ for varying DC-field current $I_{\mathrm{r} 2 \mathrm{~d}}$. All other current setpoints are 0 . Full lines are obtained via FE calculations while the stars are based on measurements. The considered EVT in this figure is the reference EVT.

\section{GenerAL SCALING LAWS}

\section{A. Theoretical background}

Scaling laws have been used in the past to provide a prediction of the average specific torque of a scaled machine based on a reference machine. The advantage of these scaling laws is the simplicity of the method and the fact that they can be applied at an early design stage. Examples can be found in literature for switched reluctance machines [17], induction machines [18] and interior permanent magnet machines [19]. The work presented in [17] has later been used by Stipetic [11] to derive scaling laws for a PMSM which are used in this paper for a PM-EVT.

There are 3 scaling parameters: rewinding $K_{\mathrm{W}}$, axial scaling $K_{\mathrm{A}}$ and radial scaling $K_{\mathrm{R}}$. Rewinding is done to meet the 
voltage requirements of the power supply system [11] and has no impact on the dimensions of the scaled EVT. Changing $K_{\mathrm{W}}$ is done by modifying the number of turns per coil $N_{\mathrm{c}}$, the number of parallel paths $a_{\mathrm{p}}$ or a combination of both:

$$
K_{\mathrm{W}}=\frac{a_{\mathrm{p}, 0}}{a_{\mathrm{p}}} \frac{N_{\mathrm{c}}}{N_{\mathrm{c}, 0}}
$$

in which subscript 0 is used to refer to the reference machine. Axial scaling $K_{\mathrm{A}}$ is carried out by varying the axial core length $l_{\mathrm{a}}$ while the lamination cross-section is preserved:

$$
K_{\mathrm{A}}=\frac{l_{\mathrm{a}}}{l_{\mathrm{a}, 0}}
$$

Radial scaling considers a proportional change of all dimensions of the cross-section. The radial scaling factor $K_{\mathrm{R}}$ is therefore equal to the ratio of the diameter of the scaled $D$ and reference $D_{0}$ EVT respectively:

$$
K_{\mathrm{R}}=\frac{D}{D_{0}}
$$

As reported by [11], the presented method is valid for any PM machine. Therefore, the objective of this section is to check whether the scaling laws are still applicable if a PMEVT is considered.

According to [11], the scaling laws are only valid if the magnetic flux densities in the core of the scaled machine are preserved. To find the conditions for which this statement holds, the Poisson's equation is written for the reference machine [11]:

$$
\frac{\partial}{\partial x}\left(\frac{1}{\mu} \frac{\partial A_{\mathrm{z}}}{\partial x}\right)+\frac{\partial}{\partial y}\left(\frac{1}{\mu} \frac{\partial A_{\mathrm{z}}}{\partial y}\right)=-J_{\mathrm{z}}
$$

and the scaled machine:

$$
\frac{\partial}{\partial x, 0}\left(\frac{1}{\mu, 0} \frac{\partial A_{z, 0}}{\partial x, 0}\right)+\frac{\partial}{\partial y, 0}\left(\frac{1}{\mu, 0} \frac{\partial A_{z, 0}}{\partial y, 0}\right)=-J_{z, 0}
$$

in which the slot current density scales with $K_{\mathrm{J}}, A_{\mathrm{z}, 0}$ and $A_{\mathrm{z}}$ resemble the cross-sectional surface of the reference and the scaled machine respectively, and $x \& y$ are dimensions which scale with the radial scaling factor $K_{\mathrm{R}}$. In order to preserve the magnetic flux density, i.e. the value of $\mu$, the terms in the parentheses must be equal for both the reference and scaled machine [11].

$$
\frac{1}{K_{\mathrm{R}}} \frac{\partial}{\partial x}\left(\frac{1}{\mu} \frac{\partial A_{\mathrm{z}}}{\partial x}\right)+\frac{1}{K_{\mathrm{R}}} \frac{\partial}{\partial y}\left(\frac{1}{\mu} \frac{\partial A_{\mathrm{z}}}{\partial y}\right)=-K_{\mathrm{J}} J_{\mathrm{z}}
$$

This is true if:

$$
K_{\mathrm{J}}=\frac{1}{K_{\mathrm{R}}}
$$

As the cross-section scales with $K_{\mathrm{R}}^{2}$, the scaled current will be proportional to the radial scaling factor. When combined with the winding factor (see [11]), the current in the scaled machine should meet the following equation:

$$
I=\frac{K_{\mathrm{R}}}{K_{\mathrm{W}}} I_{0}
$$

with $I$ an array composed of the 5 currents which flow in the scaled machine, $K_{\mathrm{R}}$ the radial scaling factor, $K_{\mathrm{W}}$ the rewinding factor and $I_{0}$ an array of currents flowing in the reference machine. If the relation in (10) is met, then the flux linkage in the machine will scale as [11]:

$$
\Psi(I)=K_{\mathrm{A}} K_{\mathrm{R}} K_{\mathrm{W}} \Psi_{0}\left(I_{0}\right)
$$

Based on (11), it is now possible to derive the scaling law for the generated torque:

$$
T=\frac{3}{2} N_{\mathrm{p}}\left(\Psi_{\mathrm{q}} I_{\mathrm{d}}-\Psi_{\mathrm{d}} I_{\mathrm{q}}\right)
$$

which can be rewritten in terms of the reference EVT based on (10) and (11):

$$
\begin{aligned}
T & =\frac{3}{2} N_{\mathrm{p}}\left(K_{\mathrm{A}} K_{\mathrm{R}} K_{\mathrm{W}} \Psi_{\mathrm{q}, 0} \frac{I_{\mathrm{d}, 0} K_{\mathrm{R}}}{K_{\mathrm{W}}}\right. \\
& \left.-K_{\mathrm{A}} K_{\mathrm{R}} K_{\mathrm{W}} \Psi_{\mathrm{d}, 0} \frac{I_{\mathrm{q}, 0} K_{\mathrm{R}}}{K_{\mathrm{W}}}\right) \\
& =K_{\mathrm{A}} K_{\mathrm{R}}^{2} \frac{3}{2} N_{\mathrm{p}}\left(\Psi_{\mathrm{q}, 0} I_{\mathrm{d}, 0}-\Psi_{\mathrm{d}, 0} I_{\mathrm{q}, 0}\right) \\
& =K_{\mathrm{A}} K_{\mathrm{R}}^{2} T_{0}\left(I_{0}\right) \\
& =K_{\mathrm{A}} K_{\mathrm{R}}^{2} T_{0}\left(\frac{K_{\mathrm{W}}}{K_{\mathrm{R}}} I\right)
\end{aligned}
$$

with $T=\left[T_{\mathrm{s}} T_{\mathrm{r} 2} T_{\mathrm{r} 1}\right]$ and $\left.T_{0}={ }_{\left[T_{\mathrm{s}, 0}\right.} T_{\mathrm{r} 2,0} T_{\mathrm{r} 1,0}\right]$ the electromagnetic torque of the scaled machine and the reference machine, respectively.

\section{B. Validation based on FE}

To validate the scaling law depicted in (11), a new FE based model is made for an EVT which is $20 \%$ longer and $30 \%$ wider $\left(K_{\mathrm{A}}=1.2\right.$ and $\left.K_{\mathrm{R}}=1.3\right)$. No winding scaling is applied $\left(K_{\mathrm{W}}=1\right)$.

To demonstrate how the scaling law needs to be applied, the stator flux in the $d$-axis will be calculated based on the scaling laws. The considered currents which flow in the scaled design are: $I_{\mathrm{r} 2 \mathrm{~d}}=-6.5 \mathrm{~A}, I_{\mathrm{sd}}=-50 \mathrm{~A}$, all other currents are equal to $0 \mathrm{~A}$. To calculate the flux, the following steps need to be taken:

1) Scale the currents based on (10): $I_{\mathrm{r} 2 \mathrm{~d}, 0}=-5 \mathrm{~A}, I_{\mathrm{sd}, 0}=$ $-38.46 \mathrm{~A}$.

2) Determine $\Psi_{\mathrm{sd}, 0}$ in the reference machine for these currents: -0.256 Vs (see Fig. 3 (a), red line)

3) Scale the flux according to (11): $-0.399 \mathrm{Vs}$

Fig. 4 (a) shows that the same result is found via the FE model. Moreover, the figure compares the results of all current setpoints which were originally presented in Fig. 3. The figure clearly shows that the scaling laws are valid. Furthermore, we can conclude that recalculating the current to flux relations that cover 5 dimensions is not necessary if a scaled version of the reference EVT is considered.

Besides the validation of the scaling law for flux, Fig. 4 shows that the scaling law for the generated torque is also applicable. The following example is chosen to demonstrate the method: $I_{\mathrm{r} 2 \mathrm{~d}}=-6.5 \mathrm{~A}$ and $I_{\mathrm{sq}}=130 \mathrm{~A}$, all other currents are equal to $0 \mathrm{~A}$. To calculate the torque, the following steps need to be taken: 
1) Scale the currents based on (10): $I_{\mathrm{r} 2 \mathrm{~d}, 0}=-5 \mathrm{~A}, I_{\mathrm{sq}, 0}=$ $100 \mathrm{~A}$.

2) Determine $T_{\mathrm{s}, 0}$ in the reference machine for these currents: $93 \mathrm{Nm}$ (see Fig. 3 (c), red line)

3) Scale the torque according to (13): $188.6 \mathrm{Nm}$

When this is compared with the torque calculated based on FE (red curve in Fig. 4 (c)), a torque of $188.6 \mathrm{Nm}$ is found that justifies the scaling law. A similar analysis has been carried out to analyze the back-EMF of the scaled machine. From that analysis it is concluded that the scaling law for the voltage depicted in [11] holds for a PM-EVT as well. The same conclusion holds for the power factor.

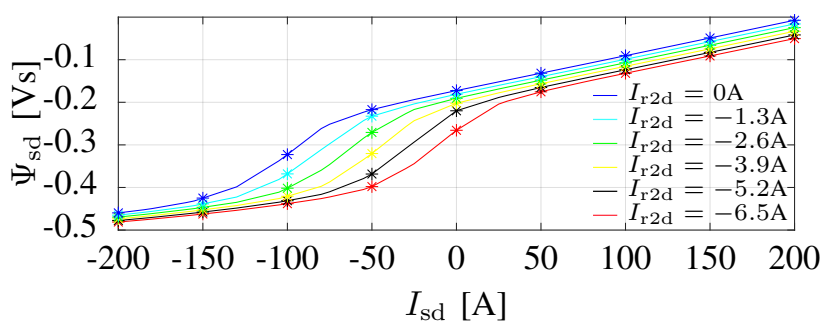

(a)

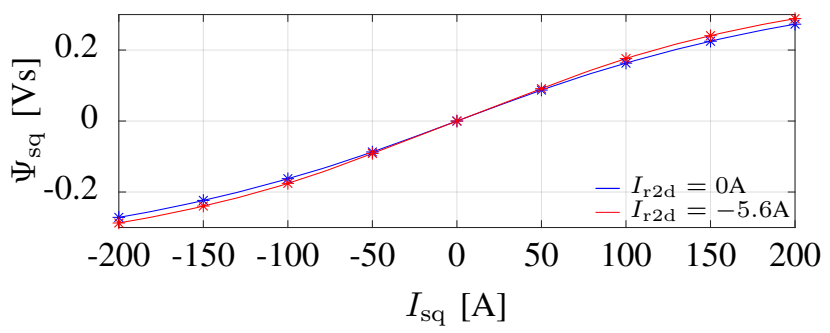

(b)

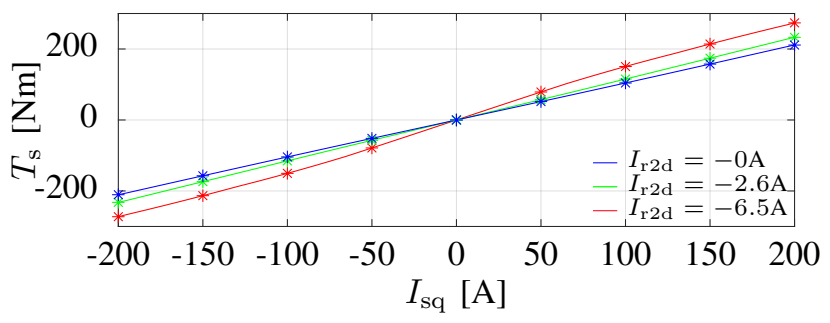

(c)

Fig. 4. (a) Stator flux in the $d$-axis $\Psi_{\text {sd }}$ as function of the stator current in the $d$-axis $I_{\mathrm{sd}}$ for varying DC-field current $I_{\mathrm{r} 2 \mathrm{~d}}$. (b) Stator flux in the $q$-axis $\Psi_{\mathrm{sq}}$ as function of the stator current in the $q$-axis $I_{\mathrm{sq}}$ for varying DC-field current $I_{\mathrm{r} 2 \mathrm{~d}}$. (c) Stator torque $T_{\mathrm{S}}$ as function of stator current in the $q$-axis $I_{\mathrm{sq}}$ for varying DC-field current $I_{\mathrm{r} 2 \mathrm{~d}}$. All other current setpoints are 0 . Full lines are obtained via FE calculations of a design characterized by a length which is 1.2 times the reference length $\left(K_{\mathrm{A}}=1.2\right)$ and a diameter which is 1.3 times the reference diameter $\left(K_{\mathrm{R}}=1.3\right)$. The stars are obtained by using the presented scaling laws.

Fig. 4 showed that the scaling laws are accurate for the considered scaling factors, i.e. $K_{\mathrm{A}}=1.2$ and $K_{\mathrm{R}}=1.3$. To demonstrate that the method stays accurate for multiple scaling factors, Fig. 5 has been added. The same conclusions can be drawn for varying axial scaling factors.

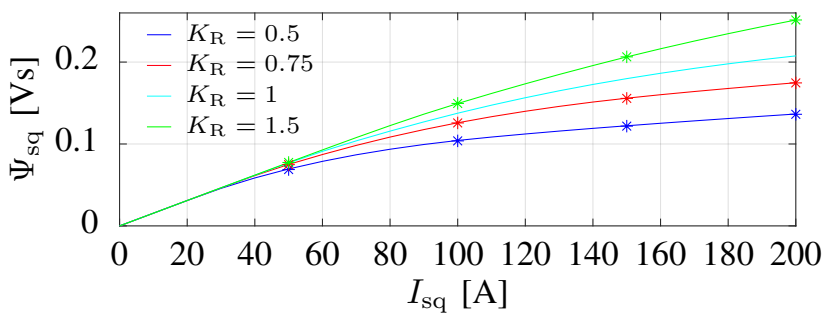

Fig. 5. Stator flux in the $q$-axis $\Psi_{\mathrm{sq}}$ as function of the stator current in the $q$-axis $I_{\mathrm{sq}}$. All other current setpoints are 0 . Full lines are obtained via FE calculations of a design characterized by the specified scaling factors $\left(K_{\mathrm{A}}=1\right.$ and $K_{\mathrm{R}}$ varies between 0.5 and 1.5). The stars are obtained by using the presented scaling laws (no scaling for the cyan curve, i.e. $K_{\mathrm{A}}=K_{\mathrm{R}}=1$ ).

\section{Validation based on literature}

In the previous subsection, the scaling laws were validated based on FE calculations. However, applying the scaling laws on an EVT prototype which is described in literature can prove the applicability of the method in real life as well. A similar methodology has been applied for PM machines used in wind generator systems [20], [21]. The EVT which is used to perform the validation is described by Pisek [7]. It is also a PM-EVT but without DC-field winding and flux bridge as shown in Fig. 1. To eliminate the behavior of this part, the current in the DC-field winding is chosen in such a way that the flux through the flux bridge is 0 . The main reasons for the choice of this paper are the comprehensive description of the geometry and the results which describe the relation between generated torque and current.

Based on the dimensions of the EVT described by Pisek it is possible to derive the following scaling factors: $K_{\mathrm{A}}=1.15$, $K_{\mathrm{R}}=0.55$ and $K_{\mathrm{W}}=1$. When these scaling factors are applied to the EVT described in this paper the results in Fig. 6 are obtained. For the inner rotor, a close match is obtained while the scaled stator torque deviates from the measured torque presented in [7] at higher currents. As the relation between current and torque is strictly linear in the case of the Pisek EVT, the saturation level remains constant. This could be due to the perforation in the outer rotor which is likely to be saturated while the field in the stator yoke remains limited. In contrast to the Pisek EVT, the stator yoke and teeth of the EVT presented in this paper are saturated for higher currents which results in lower torque as shown in Fig. 6 (b).

\section{SCALABLE OPTIMAL CONTROL}

Fig. 4 (c) shows that an infinite number of current combinations will result in the same generated torque. If a stator torque of $200 \mathrm{Nm}$ and inner rotor torque of $0 \mathrm{Nm}$ are considered, then the green line shown in Fig. 4 (c) offers the lowest losses (see Table III). However, if the optimal set of currents is searched for via the algorithm described in [12], it is shown that the losses can be further reduced with $30 \%$. It is thus not enough to scale the current to flux relations based on (11), the control needs to be scaled as well. To establish this optimal control, the optimal set of currents resulting in the desired OP, need to be found. As mentioned in the introduction, this OP is defined 


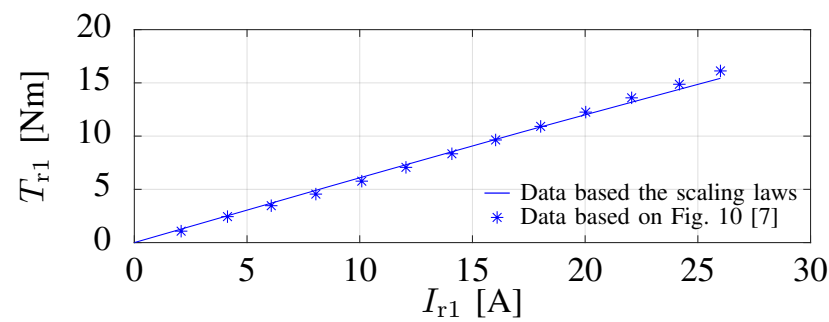

(a)

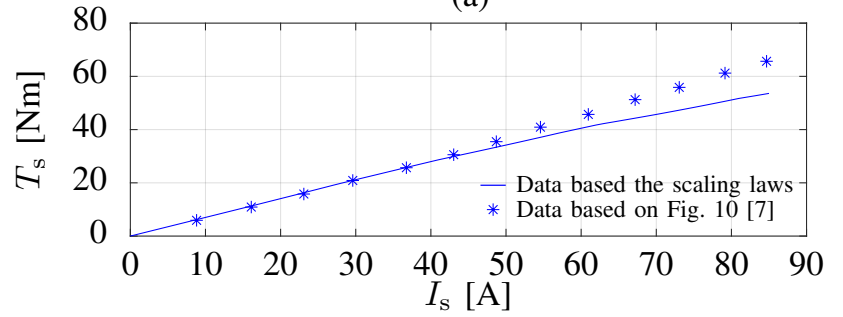

(b)

Fig. 6. Comparison between measured and scaled torque for the inner rotor (a) and stator (b) respectively. The measure torque values are obtained via [7] in Fig. 10 and 11 respectively.

TABLE III

CURRENT SETPOINTS FOR A REFERENCE STATOR TORQUE OF 200 NM, INNER ROTOR TORQUE OF $O$ NM, INNER ROTOR SPEED OF 0 RPM AND AN OUTER ROTOR SPEED OF 1000 RPM.

\begin{tabular}{|l|l|l|l|l|}
\cline { 2 - 5 } \multicolumn{1}{c|}{} & Blue & Green & Red & Optimal \\
\hline$I_{\mathrm{Sq}}[\mathrm{A}]$ & 189.4 & 172 & 138.9 & -133.6 \\
\hline$I_{\mathrm{sd}}[\mathrm{A}]$ & 0 & 0 & 0 & 79.6 \\
\hline$I_{\mathrm{r} 2 \mathrm{~d}}[\mathrm{~A}]$ & 0 & -2.6 & -6.5 & -1.1 \\
\hline$I_{\mathrm{r} 1 \mathrm{q}}[\mathrm{A}]$ & 0 & 0 & 0 & -0.4 \\
\hline$I_{\mathrm{r} 1 \mathrm{~d}}[\mathrm{~A}]$ & 0 & 0 & 0 & -14.5 \\
\hline$P_{\mathrm{cu}}[\mathrm{W}]$ & 886 & 803 & 926 & 570 \\
\hline$P_{\mathrm{fe}}[\mathrm{W}]$ & 357 & 353 & 340 & 238 \\
\hline$P_{\text {loss }}[\mathrm{W}]$ & 1243 & 1156 & 1266 & 808 \\
\hline
\end{tabular}

by the torque developed by the inner rotor and stator and the speed of both rotors.

The optimization routine, of which the results have been validated on a prototype, can be written as in [12]:

Find:

$$
I^{*}=\left[I_{\mathrm{sq}}^{*}, I_{\mathrm{sd}}^{*}, i_{\mathrm{r} 2 \mathrm{~d}}^{*}, I_{\mathrm{r} 1 \mathrm{q}}^{*}, I_{\mathrm{r} 1 \mathrm{~d}}^{*}\right] \in \mathbb{R}^{5}
$$

with:

$$
I^{*}=\arg \min \left(P_{\text {loss }}\left(I, \omega_{\mathrm{r} 1}, \omega_{\mathrm{r} 2}\right)\right)
$$

subjected to:

$$
T_{\mathrm{s}}\left(I^{*}\right)=T_{\mathrm{s}, \mathrm{set}} \& T_{\mathrm{r} 1}\left(I^{*}\right)=T_{\mathrm{r} 1, \mathrm{set}}
$$

The generated torque is calculated based on (1) and (2), $P_{\text {loss }}$ is equal to the sum of iron and copper losses. As solving this routine takes some time, the optimization is carried out offline after which the results are stored in a Look-Up Table (LUT) which is consulted during operation. The LUT thus basically yields the optimal current setpoints given an OP defined by a stator $T_{\mathrm{s}}$ and inner rotor $T_{\mathrm{r} 1}$ torque setpoint and inner and outer rotor speed denoted by $N_{\mathrm{r} 1}$ and $N_{\mathrm{r} 2}$ respectively. Of course, it is not feasible to have to repeat this optimization routine for every new design. Therefore, the following paragraphs will discuss the scalable control. As a result, the time-consuming method as elaborated in [12] will only have to be computed once, i.e. for the reference EVT.

Scalable control has recently been developed to scale the efficiency map of a PMSM [14], [22]. In these papers, MTPA and FW algorithms in fmincon functions (nonlinear solver in Matlab $(R)$ are used to determine the optimal currents of the scaled machine based on the reference machine and its LUTs. However, to the knowledge of the authors, this has not been discussed in literature for a PM-EVT so far. Therefore, compared to the State-of-the-Art, this scalable control for an EVT is a new and crucial step in the scaling law based sizing methodology of an EVT.

The first step in obtaining the desired scalable control is to scale the torque setpoint for the OP at the input side of the LUT containing the optimal control of the reference EVT (see Fig. 7). Hence, the second step is to scale the current back towards the scaled EVT based on (10). Validation of this scalable optimal control is done on the earlier introduced reference EVT and the rescaled EVT. The results of this methodology are shown in Fig. 8. The continuous lines show the actual optimal currents for the scaled EVT which are determined based on the method presented in [12] while the stars represent the results based on the scalable optimal control. The figure shows that there is only a minimal difference between the currents that are acquired via both methods. Moreover, the difference in losses is negligible (see Fig. 8 (d)). Note that the inner rotor current in the $d$-axis is close to zero. This is because the inner rotor has no saliency, $I_{\mathrm{r} 1 \mathrm{~d}}$ will thus have no effect on the torque, but will only increase the copper losses.

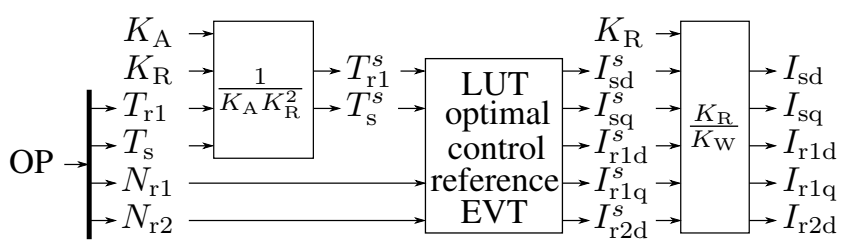

Fig. 7. Methodology to obtain the optimal control for a scaled EVT based on the optimal control of the reference EVT.

\section{SIZING METHODOLOGY}

The proposed sizing methodology uses the elaborated scaling laws and the scalable optimal control to graphically assess the feasibility of a design. Feasibility is defined by the losses of the design and whether the design fits the demanded constraints such as torque or weight limitations. To graphically combine all this information, a performance map is used. This map shows the losses $P_{\text {loss }}$, composed of copper and iron losses, as a function of the axial and radial scaling factor for a given OP. The following subsections provide a method to set up a performance map and demonstrate how limitations can be visualized. 


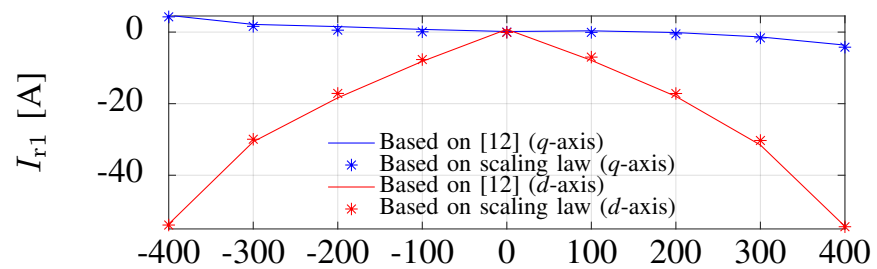

(a)

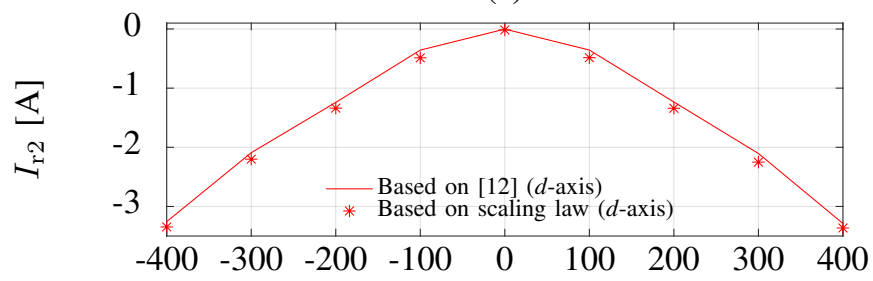

(b)

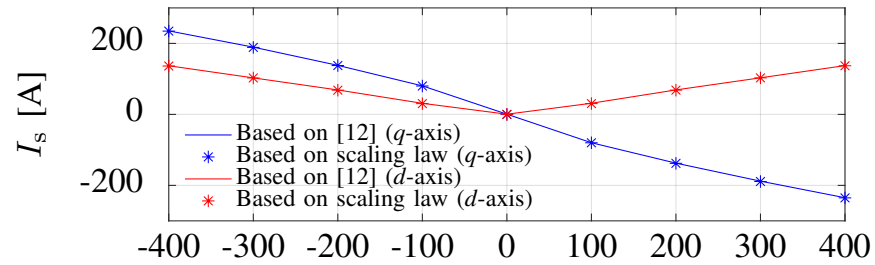

(c)

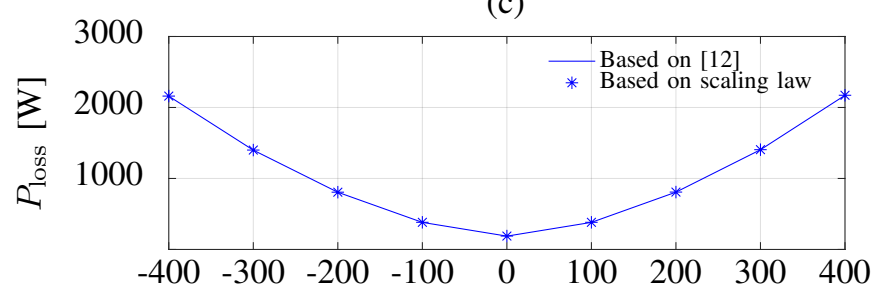

(d)

$T_{\mathrm{s}}[\mathrm{Nm}]$

Fig. 8. (a)-(c) Optimal currents for varying stator torque $T_{\mathrm{S}}$ and inner rotor torque $T_{\mathrm{r} 1}$ of $0 \mathrm{Nm}$. (d) Losses for the calculated optimal current set. The speed of the inner rotor $N_{\mathrm{r} 1}$ is $0 \mathrm{rpm}$ while the outer rotor speed $N_{\text {r2 } 2}$ is $1000 \mathrm{rpm}$.

\section{A. Step 1: performance map boundaries and design con- straints}

The first step in setting up the performance map is to define the boundaries and stepsize for the considered scaling factors. This results in a grid of axial and radial scaling factors which characterize the considered designs (see Fig. 9). Note that a third axis can be added for the winding factor. In the remainder of the paper, the winding factor will be set to 1 .

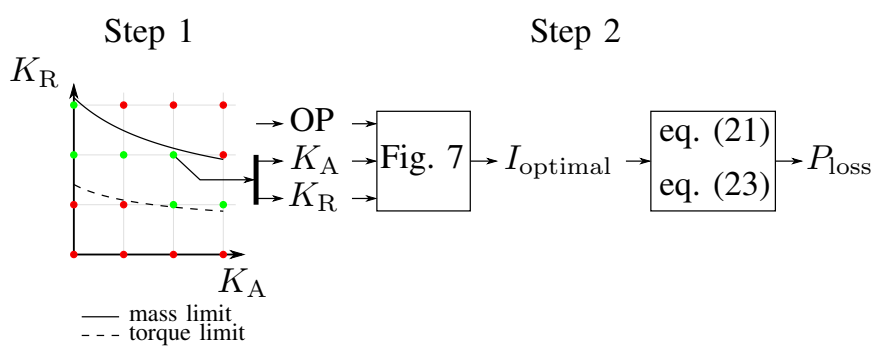

Fig. 9. Necessary steps in setting up a performance map. Step 1: define a grid of possible designs and add the constraints. Step 2: determine the losses for each design that fits the constraints.
The grid thus defines a set of designs for which the losses could be determined (visualized by the green and red dots in Fig. 9). As not all combinations of $K_{\mathrm{A}}$ and $K_{\mathrm{R}}$ are realistic, it is essential to add constraints, at least concerning weight and required torque. Those constraints are the solid and dashed line in Fig. 9, respectively. The applicable range for the scaling factors is restricted due to thermal and technological reasons. However, if an axial and radial scaling factor of 1.25 is chosen, the output torque will already be approximately $200 \%$ higher [22].

To determine the maximum torque limit, the force density is used. The force density $F_{\mathrm{d}}$ is a measure for the force developed per square meter of an active air gap surface area. This parameter is rather constant over a wide range of machine power values [23], [24]. This makes the force density particularly suitable to estimate the torque boundaries for designs with varying power ranges. The maximum torque $T_{\max }$ (inner rotor or stator torque) can be calculated as:

$$
T_{\max }=2 \pi r^{2} l_{\mathrm{a}} F_{\mathrm{d}}
$$

in which $r$ is the airgap radius, $l_{\mathrm{a}}$ is the active length and $F_{\mathrm{d}}$ is the force density. The value for $F_{\mathrm{d}}$ can be theoretically obtained as elaborated in [25], [26] or can be based on measurements. Measuring $F_{\mathrm{d}}$ comes down to determining the maximum continuous torque of the EVT for which there is still an acceptable thermal equilibrium.

As the required torque is typically one of the design criteria and thus a known property, it is more useful to relate the torque to the chosen radius of the stator or inner rotor:

$$
r=\sqrt{\frac{T_{\max }}{2 \pi F_{\mathrm{d}} l_{\mathrm{a}}}}
$$

Based on the definitions of the scaling laws, (4) and (5), it is possible to rewrite (18) as:

$$
\begin{gathered}
K_{\mathrm{R}} r_{0}=\sqrt{\frac{T_{\max }}{2 \pi F_{\mathrm{d}} K_{\mathrm{A}} l_{\mathrm{a}, 0}}} \\
K_{\mathrm{R}}=\frac{1}{r_{0}} \sqrt{\frac{T_{\max }}{2 \pi F_{\mathrm{d}} K_{\mathrm{A}} l_{\mathrm{a}, 0}}}
\end{gathered}
$$

in which $r_{0}$ is the airgap radius and $l_{\mathrm{a}, 0}$ is the active length, both of the reference machine. Equation (20) can now be used to calculate the relation between the axial and radial scaling factors given a maximum required torque $T_{\max }$ (defined by the considered application). Fig. 10 shows this relation for several torque values (dotted lines). Any design, defined by a given $K_{\mathrm{A}}$ and $K_{\mathrm{R}}$ value, which is located under the design line, will be overloaded during operation. In this paper, this design torque equals the rms value of the load profile of the considered application. However, if the load torque is not periodic, which is for example the case when HEVs are considered, the average torque could be used.

As stated at the beginning of this subsection, there are other restrictions which could be important as well. Such constraints are related to maximum weight or volume which is for example very important in HEVs. Based on the sizing 


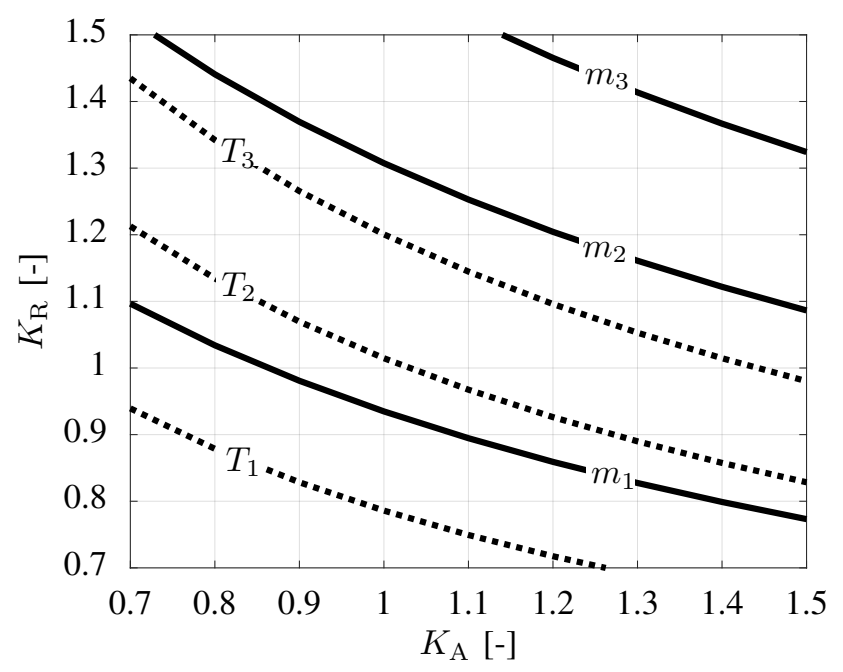

Fig. 10. Any solution below the design lines for maximum stator torque $\left(T_{1}=150 \mathrm{Nm}, T_{2}=250 \mathrm{Nm}\right.$ and $\left.T_{3}=350 \mathrm{Nm}\right)$ will result in overload for the specified torque. Solutions above the design lines for maximum mass $\left(m_{1}=50 \mathrm{~kg}, m_{2}=100 \mathrm{~kg}\right.$ and $m_{3}=150 \mathrm{~kg}$ ) leads to a heavier solution than the specified mass.

of the EVT, it is possible to calculate boundary conditions for these quantities as shown in Fig. 10 for the weight (full lines). Any design above the line will be heavier than the specified maximum value.

It is thus possible to visually analyze which designs in the considered set can be excluded based on the specified design constraints. This simplifies the design problem as shown in Fig. 9 where the designs which meet the constraints are colored green and the design that do not meet the constraints are colored red.

\section{B. Step 2: optimal currents and losses}

Step 2, as demonstrated in Fig. 9, is to determine the optimal current set for a design at a specific OP. Note that only one OP is considered per performance map. However, it is possible to integrate the losses of multiple performance maps over time if one wants to analyze a cycle (see section VI). The method for a single OP is summarized in Fig. 7. Once the currents are known, the copper losses are easily determined:

$$
P_{\mathrm{cu}}=\frac{3}{2} R_{\mathrm{s}}\left(I_{\mathrm{sd}}^{2}+I_{\mathrm{sq}}^{2}\right)+R_{\mathrm{r} 2} I_{\mathrm{r} 2 \mathrm{~d}}^{2}+\frac{3}{2} R_{\mathrm{r} 1}\left(I_{\mathrm{r} 1 \mathrm{~d}}^{2}+I_{\mathrm{r} 1 \mathrm{q}}^{2}\right)
$$

Note that the resistance of the inner rotor, outer rotor and stator scale as well:

$$
R=\frac{K_{\mathrm{W}}^{2}}{K_{\mathrm{R}}^{2}}\left(K_{\mathrm{A}} R_{\mathrm{co}, 0}+K_{\mathrm{R}} R_{\mathrm{ew}, 0}\right)
$$

in which $R_{\mathrm{co}, 0}$ is the resistance of the reference EVT in the core region, while $R_{\mathrm{ew}, 0}$ is the resistance of the end winding. In addition to the copper losses, the iron losses need to be determined. Assuming a sinusoidal air gap induction waveform, it is possible to calculate the iron losses in [W/kg] based on (23) with $a, b, c, d$ and $\alpha$ magnetic core material parameters, $\hat{B}$ the amplitude of the magnetic flux density in
TABLE IV

COMPARISON OF THE COMPUTATIONAL EFFORT TO ANALYZE 10000 OP OF A SCALED EVT.

\begin{tabular}{|l|l|l|}
\cline { 2 - 3 } \multicolumn{1}{c|}{} & $\begin{array}{l}\text { Computational effort } \\
\text { (FE calculations) [s] }\end{array}$ & $\begin{array}{l}\text { Computational effort } \\
\text { (scaling laws) [s] }\end{array}$ \\
\hline Current to flux & 350000 & 200 \\
\hline Optimal control & 60000 & $\left.{ }^{*}\right)$ \\
\hline Total & 410000 & 200 \\
\hline
\end{tabular}

(*) Happens simultaneous with the calculation

of the current to flux relations.

the iron and $f$ the frequency [27]. The values for the material properties are determined via measurements.

$$
P_{\mathrm{fe}}(t)=\left[a \hat{B}^{\alpha} f+b \hat{B}^{2} f^{2} c \hat{B} f(\sqrt{1+d \hat{B} f}-1)\right]
$$

As the losses are determined, the procedure above is repeated for every design in the grid which meets the criteria, i.e. green dots. For a detailed analysis of the separation of the losses, the authors refer to [15].

\section{Computational effort of FE vs. scaling laws}

One of the main advantages of the scaling laws is the sheer drop in the computational effort compared to FE calculations. If the scaling laws are applied, an OP of a scaled design is evaluated $0.02 \mathrm{~s}$ (Core i7 processor, $2.9 \mathrm{GHz}$ ). As already stated in section II, it takes $2 \mathrm{~s}$ per FE calculation and approximately $175000 \mathrm{FE}$ calculations are necessary to take all the current to flux relations correctly into account. This results in a calculation time of roughly 4 days. The next step is to search for the optimal control. This takes $6 \mathrm{~s}$ per $\mathrm{OP}$, which seems negligible. However, the more detail that is required from the load pattern (higher number of considered $\mathrm{OP})$, the more significant this part will become. Table IV gives an additional example when 10000 OP are considered (10 possible values for inner rotor torque, stator torque, inner rotor speed and outer rotor speed).

\section{CASE STUDY}

To demonstrate the method, a generic load profile as depicted in Table $\mathrm{V}$ has been chosen. This profile is periodically executed (OP (1) starts again after OP (4) is finished). Here, an additional constraint is a maximum weight of $200 \mathrm{~kg}$. Note that the optimization routine will result in a design which is able to execute the load cycle from an electromagnetic point of view with minimal losses. However, the thermal aspect is not considered and is out of scope for this publication as, to the knowledge of the authors, no scalable thermal model for this type of PM-EVT exist.

The first step is again to define a grid and to visually represent the limitations on the performance map. All designs which are above the mass limit or below the torque limit do not need to be considered which reduces the computational effort.

To determine the maximum torque line, the force density of the reference EVT and the rms torque of the profile need to be known. To obtain the force density, the maximum torque 
TABLE V

GENERIC LOAD PROFILE

\begin{tabular}{|c|c|c|c|c|c|}
\hline $\begin{array}{c}\text { Operating } \\
\text { point }\end{array}$ & $\begin{array}{c}T_{\mathrm{s}} \\
{[\mathrm{Nm}]}\end{array}$ & $\begin{array}{c}T_{\mathrm{r} 1} \\
{[\mathrm{Nm}]}\end{array}$ & $\begin{array}{c}N_{\mathrm{r} 1} \\
{[\mathrm{rpm}]}\end{array}$ & $\begin{array}{c}N_{\mathrm{r} 2} \\
{[\mathrm{rm}]}\end{array}$ & $\begin{array}{c}\Delta t \\
{[\%]}\end{array}$ \\
\hline (1) & 150 & 80 & 1000 & 2000 & 30 \\
\hline (2) & 200 & 20 & 750 & 2000 & 10 \\
\hline (3) & 50 & 70 & 500 & 1000 & 40 \\
\hline (4) & 80 & 50 & 750 & 1000 & 20 \\
\hline
\end{tabular}

on stator and inner rotor are considered. For the reference EVT, these maximum torque values are $245 \mathrm{Nm}$ and $137 \mathrm{Nm}$ respectively. Given the geometrical parameters of the EVT, this translates to a force density of $29.1 \mathrm{kN} / \mathrm{mm}^{2}$ for the stator and $23.9 \mathrm{kN} / \mathrm{mm}^{2}$ for the inner rotor. The difference in force density between the stator and inner rotor is related to the type of cooling. The inner rotor is cooled with air while the stator is cooled via a water jacket allowing a higher current density and thus a higher torque. The rms torque of the profile is calculated based on Table $\mathrm{V}$ and equals $134.2 \mathrm{Nm}$ for the stator and $66.4 \mathrm{Nm}$ for the inner rotor. The stator torque is thus more critical and will be taken into account for the maximum torque line. The mass limitation is purely related to geometry (which needs to be scaled) and mass density values of the used materials. Details considering the scalable geometrical model are considered out of scope for this publication.

The second step is to calculate the losses for each OP for a grid defined by axial and radial scaling factors. Based on these losses the total energy consumption for one cycle can be obtained by integrating the losses (see Fig. 11). The limitations based on the stator torque of $134.2 \mathrm{Nm}$ (dashed line Fig. 11) and a maximum mass of $200 \mathrm{~kg}$ (full line Fig. 11) are plotted on this graph to eliminate designs which do not match with the constraints. The white regions in Fig. 11 represent designs which are not considered (below the dashed line or above the full line) or which do not suffice (not acceptable although within the constraints). These insufficient designs are related to OP (2) for which a rather high torque is demanded in comparison with the rms torque for which the application is designed. The white area above the torque limit in Fig. 11 thus represents designs which are able to produce the rms torque but not the peak torque related to OP (2). As the optimal design is defined as the design with the lowest energy consumption, the minimum of the values shown in the performance map is the optimal design (see yellow circle Fig. 11).

In the previous paragraphs, a method is demonstrated to size the EVT based on constraints. However, it is also interesting to investigate the impact of those constraints on the optimal design. It is, for example, possible to investigate how the energy consumption changes as a function of the maximum considered weight, which is an important feature in automotive applications. The torque limit is less suited for this as the torque is linked with the load profile which usually cannot be changed easily.

Fig. 12 shows how the energy consumption $\Delta E$ changes as the restriction on the allowed weight changes. To set up this figure, the demonstrated method in this section has to be repeated for a range of mass values. Each of them will result in a different solution with different losses and thus energy

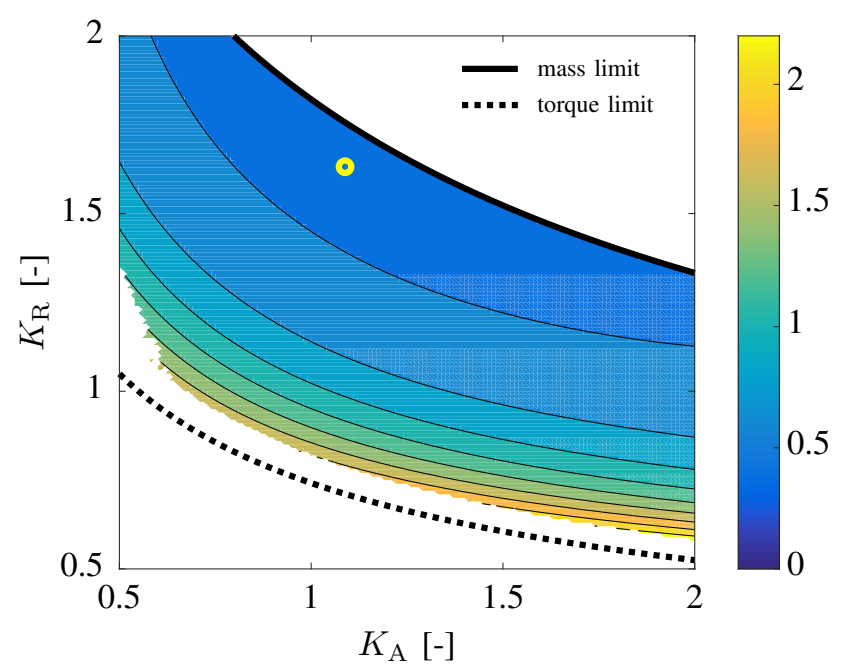

Fig. 11. Performance map displaying the energy consumption [kJ] over a cycle. The period of one cycle has been arbitrarily chosen equal to 1 second. The optimal design is highlighted as the yellow circle.

consumption. The red dot on the figure clearly shows that, for the given load cycle, it is possible to design an EVT which is roughly $50 \mathrm{~kg}$ lighter than the optimum without having a significant increase in the energy consumption (an increase of $0.7 \%$ ). This is valuable information as less weight can reduce losses at system level as well (for example in a HEV). Note that if the weight needs to reduce drastically, for example towards $100 \mathrm{~kg}$, then the energy consumption will increase by $15.2 \%$.

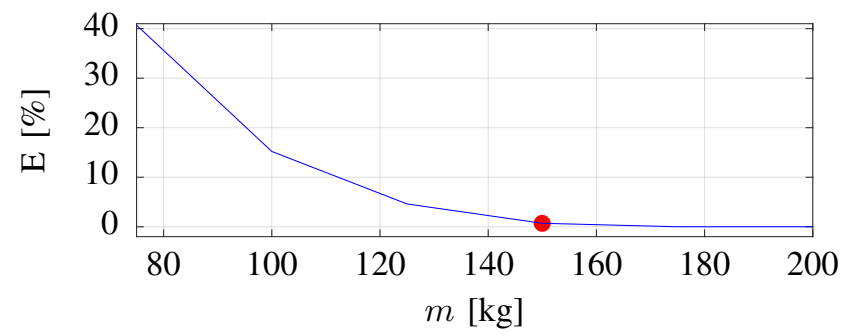

Fig. 12. Difference in energy consumption between optimal solutions for varying mass with as a reference the optimal solution at a mass of $200 \mathrm{~kg}$.

\section{CONCLUSION}

A methodology is presented to design the EVT and its control by using a reference EVT and scaling laws for the flux linkage, torque and control algorithm. Based on these scaling laws and an accurate description of the influence of the 5 independent current components on the flux linkage with the stator and inner rotor of a reference machine, it is possible to calculate copper and iron losses of a scaled EVT in a $0.02 \mathrm{~s}$. This allows for a fast assessment of design modifications via axial and radial scaling in terms of losses, maximum torque and weight, volume or inertia. All this information is combined on a single performance map. Because the performance map shows both losses and limits, 
it can be used easily to visually select the optimal design for a specific OP or a load cycle. Whether this load cycle comes from a highly dynamic application, such as a weaving loom or a less dynamically demanding vehicle application, does not have an impact on the applicability of the method. The method can thus be used as part of a general optimization routine, which selects the optimal components of a drivetrain with an EVT. As an EVT is developed for HEV applications, the primary use of the presented method is in the optimization of such drivetrains. Future developments will thus mainly focus on applying the method in optimization strategies. The main limitation of the method is that it can only optimize the overall dimensions and thus not details such as the width of the teeth. The performance of the scaled machine depends thus on how well the electromagnetic design of the reference EVT is done.

\section{REFERENCES}

[1] X. Sun, M. Cheng, W. Hua, and L. Xu, "Optimal design of doublelayer permanent magnet dual mechanical port machine for wind power application," IEEE Transactions on Magnetics, vol. 45, no. 10, pp. 46134616, 2009.

[2] X. Sun, M. Cheng, Y. Zhu, and L. Xu, "Application of electrical variable transmission in wind power generation system," IEEE Energy Conversion Congress and Exposition, vol. 49, no. 3, pp. 1529-1536, 2010.

[3] G. Carbone, L. Mangialardi, and G. Mantriota, "Fuel Consumption of a Mid Class Vehicle with Infinitely Variable Transmission," in SAE Technical Papers, pp. 2474-2484, 2001.

[4] F. Verbelen, J. Druant, S. Derammelaere, F. De Belie, K. Stockman, and P. Sergeant, "Benchmarking the permanent magnet electrical variable transmission against the half toroidal continuously variable transmission," Mechanism and Machine Theory, vol. 113, pp. 141-157, 2017.

[5] J. Druant, H. Vansompel, F. De Belie, and P. Sergeant, "Efficiency of a CVT Operated EVT Experimentally Evaluated against Half-Toroidal and Push-belt CVTs," IEEE Transactions on Industrial Electronics, vol. 65, no. 4, pp. 3095-3103, 2018.

[6] J. Druant, H. Vansompel, F. De Belie, J. Melkebeek, and P. Sergeant, "Torque Analysis on a Double Rotor Electrical Variable Transmission With Hybrid Excitation," IEEE Transactions on Industrial Electronics, vol. 64, no. 1, pp. 60-68, 2017.

[7] P. Pisek, B. Stumberger, T. Marcic, and P. Virtic, "Design analysis and experimental validation of a double rotor synchronous PM machine used for HEV," IEEE Transactions on Magnetics, vol. 49, no. 1, pp. 152-155, 2013.

[8] Y. Cheng, C. Espanet, R. Trigui, A. Bouscayrol, and S. Cui, "Design of a permanent magnet electric variable transmission for HEV applications," 2010 IEEE Vehicle Power and Propulsion Conference, 2010.

[9] Y. Cheng, R. Trigui, C. Espanet, and A. Bouscayrol, "Specifications and Design of a PM Electric Variable Transmission for Toyota Prius II," IEEE Transactions on vehicular technology, vol. 60, no. 9, pp. 41064114, 2011.

[10] G. R. Slemon, "On the design of high-performance surface-mounted PM motors," IEEE Transactions on Industry Applications, vol. 30, no. 1, pp. 134-140, 1993.

[11] S. Stipetic, D. Zarko, and M. Popescu, "Ultra-fast axial and radial scaling of synchronous permanent magnet machines," IET Electric Power Applications, vol. 10, no. 7, pp. 658-666, 2016.

[12] J. Druant, H. Vansompel, F. De Belie, and P. Sergeant, "Optimal Control for a Hybrid Excited Dual Mechanical Port Electric Machine," IEEE Transactions on Energy Conversion, vol. 32, no. 2, pp. 599-607, 2017.

[13] E. A. Grunditz and T. Thiringer, "Electric Vehicle Acceleration Performance and Motor Drive Cycle Energy Efficiency Trade-Off," in 2018 XIII International Conference on Electrical Machines (ICEM), pp. 717 $723,2018$.

[14] K. Ramakrishnan, S. Stipetic, M. Gobbi, and G. Mastinu, "Optimal Sizing of Traction Motors Using Scalable Electric Machine Model," IEEE Transactions on Transportation Electrification, vol. 4, no. 1, pp. 314-321, 2018 .
[15] J. Druant, H. Vansompel, F. De Belie, and P. Sergeant, "Loss Identification in a Double Rotor Electrical Variable Transmission," IEEE Transactions on industrial electronics, vol. 64, no. 10, pp. 7731-7740, 2017.

[16] M. Hoeijmakers, "Rotating electromechnical converter," Patent US 9 018 863, DOI US 2010/0311130 Al, 2015.

[17] Q. S. Gu and M. Stiebler, "Scaling and Dimensioning of Switched Reluctance Machines," International Transactions on Electrical Energy Systems, vol. 7, no. 5, pp. 301-310, 1997.

[18] J. Bone, "Influence of rotor diameter and length on the rating of induction motors," IEE Journal on Electric Power Applications, vol. 1, no. 1, pp. 1-5, 1978 .

[19] D. Zarko and S. Stipetic, "Criteria for Optimal Design of Interior Permanent Magnet Motor Series," in International Conference on Electrical Machines (ICEM), pp. 1242-1249, 2012.

[20] H. Li, Z. Chen, and H. Polinder, "Optimization of multibrid permanentmagnet wind generator systems," IEEE Transactions on Energy Conversion, vol. 24, no. 1, pp. 82-92, 2009.

[21] A. McDonald, M. Mueller, and H. Polinder, "Structural mass in directdrive permanent magnet electrical generators," Renewable Power Generation, IET, vol. 1, no. 1, pp. 10-16, 2007.

[22] S. Stipetic, J. Goss, and D. Zarko, "Calculation of Efficiency Maps Using Scalable Saturated Model of Synchronous Permanent Magnet Machines,' IEEE Transactions on Industry Applications, vol. 54, no. 5, pp. 4257 - 4267, 2018.

[23] H. Polinder, S. de Haan, and M. Dubois, "Basic Operation Principles and Electrical Conversion Systems of Wind Turbines," EPE Journal, vol. 15, no. 4, pp. 43-50, 2005.

[24] J. Pyrhönen, T. Jokinen, and V. Hrabovcová, Design of Rotating Electrical Machines. John Wiley \& Sons, Ltd, 2009.

[25] A. Grauers and P. Kasinathan, "Force Density Limits in Low-Speed PM Machines Due to Temperature and Reactance," IEEE Transaction on Energy Conversion, vol. 19, no. 3, pp. 518-525, 2004.

[26] P. Kasinathan, A. Grauers, and E. S. Hamdi, "Force Density Limits in Low-Speed Permanent-Magnet Machines Due to Saturation," IEEE Transaction on Energy Conversion, vol. 20, no. 1, pp. 37-44, 2005.

[27] G. Bertotti, Hysteresis in magnetism: for physicists, materials scientists, and engineers. Academic Press, 1998.

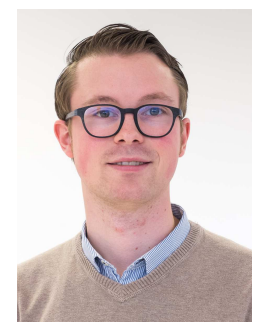

Florian Verbelen was born in Kortrijk, Belgium, in 1990. He received the Master's degree in electro mechanical engineering from the Technical University College of West Flanders, Kortrijk, Belgium, in 2013. He is currently working toward the Ph.D. degree at Ghent University, Ghent, Belgium, where he is researching the energetic and dynamic impact of variable transmissions on advanced drivetrains.

Ahmed Abdallh was born in Egypt in 1980. $\mathrm{He}$ received the B.Sc. and M.Sc. degrees in electrical engineering from Cairo University, Giza, Egypt, in 2003 and 2006, respectively. In 2012, he received the Ph.D. degree in electromechancial engineering from the University of Ghent, Gent, Belgium. From 2012 to 2015, he worked as a Postdoctoral Researcher at Ghent University. In 2015, he was a Visiting Researcher at Aalto University, Finland. He is currently working as a Research Engineer at Flanders Make (the strategic research centre for the manufacturing industry in Belgium). His research interests include numerical methods for electromagnetics, magnetic materials identification, inverse problems, electric machine design, and electrification of industrial/vehicle drivetrains.

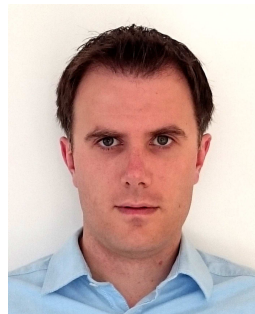

Hendrik Vansompel was born in Belgium in 1986. He received the master's and Ph.D. degrees in electromechanical engineering from Ghent University, Ghent, Belgium, in 2009 and 2013, respectively. He is currently a Postdoctoral Research Assistant with the Department of Electrical Energy, Metals, Mechanical Constructions and Systems, Ghent University. His research interests include the design and control of electrical drives. 

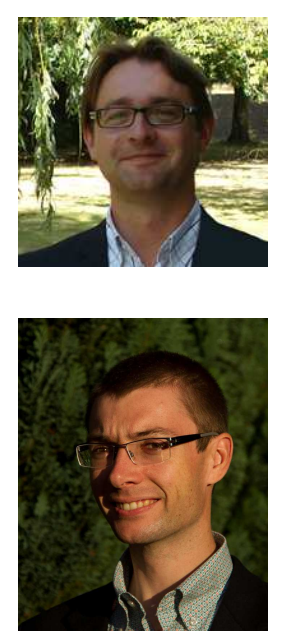

Kurt Stockman started his career at Hogeschool West-Vlaanderen in 1995. Since October 2013 he is a professor at Ghent University. During his PhD, he studied the behaviour of adjustable speed drives during voltage dips. After his PhD he concentrated more on the efficiency and control of motor driven systems. His research is characterized by a strong experimental basis and intense collaboration with industry.

Peter Sergeant received the M.Sc. degree in electromechanical engineering in 2001, and the $\mathrm{Ph} . \mathrm{D}$. degree in engineering sciences in 2006, both from Ghent University, Ghent, Belgium. In 2001, he became a researcher at the Electrical Energy Laboratory of Ghent University. He became a postdoctoral researcher at Ghent University in 2006 (postdoctoral fellow of the Research Foundation - Flanders) and at Ghent University College in 2008. Since 2012, he is associate professor at Ghent University. His current research interests include design of electrical machines with high efficiency and power density for sustainable energy applications. 